\title{
THE ORION NEBULA
}




\title{
THE STRUCTURE OF MOLECULAR CLOUDS FROM LARGE SCALE SURVEYS OF CO AND CS
}

\author{
John Bally \\ AT\&T Bell Laboratories \\ HOH-L245, Holmdel, NJ 07733
}

\begin{abstract}
The molecular gas lying in the interior of the Orion superbubble consists of sheets, filaments and bubbles in which the dense active star forming cloud cores are embedded. Many regions have a wind-swept or cometary appearance suggesting strong interaction with the Orion $\mathrm{OB}$ association. Extemal heating dominates the cloud energy budget, resulting in cold ( $10 \mathrm{~K})$ cloud cores surrounded by hot $(>20 \mathrm{~K})$ envelopes. Non-ionizing UV radiation emitted by late $\mathrm{B}$ and A stars may be the source of the kinetic energy needed to form some of the large scale cavities and contributes to the supersonic line widths seen in many clouds.
\end{abstract}

\section{INTRODUCTION}

The formation, evolution, star formation history, and disruption of molecular clouds can be studied by mapping their spatial structure and kinematics using millimeter-wave spectral lines. During the past 5 years, $70 \%$ of the observing time on the 7-meter diameter millimeter wave telescope at Crawford Hill, which has a $100^{\prime \prime}$ beam at $110 \mathrm{GHz}$, has been dedicated to the large scale mapping of molecular clouds. This effort has involved the entire Bell Labs group consisting of $R$. W. Wilson, A. A. Stark, W. D. Langer, and myself. Of the 350,000 spectra, about $85 \%$ are in the $\mathrm{J}=1-0{ }^{13} \mathrm{CO}$ line, $10 \%$ in the $\mathrm{J}=2-1 \mathrm{CS}$ line, and $5 \%$ in the $\mathrm{J}=1-0{ }^{12} \mathrm{CO}$ line. In the Orion region alone, about 180,000 spectra have been obtained. Since each spectrum consists of over 60 independent velocity channels, the display and analysis of this data presents a major challenge. One approach is to utilize a three color display in which the line intensity in three adjacent narrow velocity ranges are simultaneously displayed by the red, green, and blue guns of an RGB monitor. This method renders small scale fluctuations in the velocity field as true color and presents the line intensity as the brightness of the display.

\section{THE LARGE SCALE STRUCTURE OF THE ORION REGION}

Orion contains one of the nearest sites of active massive star formation, located at a distance of about $500 \mathrm{pc}$. Over the last $10^{7}$ years, an $\mathrm{OB}$ association with at least several dozen O stars and many more B and later type stars has formed (see Goudis 1982 for a review of early work). A large 10 by 25 degree diameter bubble surrounding this region is visible in optical emission lines (Sivan 1974). Barnard's Loop is located at the eastern edge of the bubble and a large " $U$ "-shaped emission nebula in Eridanus marks its western edge. Faint emission connecting these features may represent the outer boundary of a "superbubble" blown in the interstellar medium by the combined effect of ionizing radiation, stellar winds, and supernova explosions over the past $10^{7}$ years (Cowie, Songaila, and York 1979). Most of the interior may be filled with hot $\left(10^{5}\right.$ to $\left.10^{6} \mathrm{~K}\right)$ gas. The oblong shape of the bubble is probably produced by the decreasing pressure gradient toward high galactic latitudes. At its high latitude end (in Eridanus) the bubble may have "burst", pumping hot gas into the galactic corona, making Orion the nearest example of a "galactic fountain" (Bregman 1980).

Two giant molecular clouds reside in the interior of the Orion bubble. The Orion $\mathrm{A}$ cloud in the south contains the HII regions M42 (Orion Nebula) and NGC1977. The Orion B 


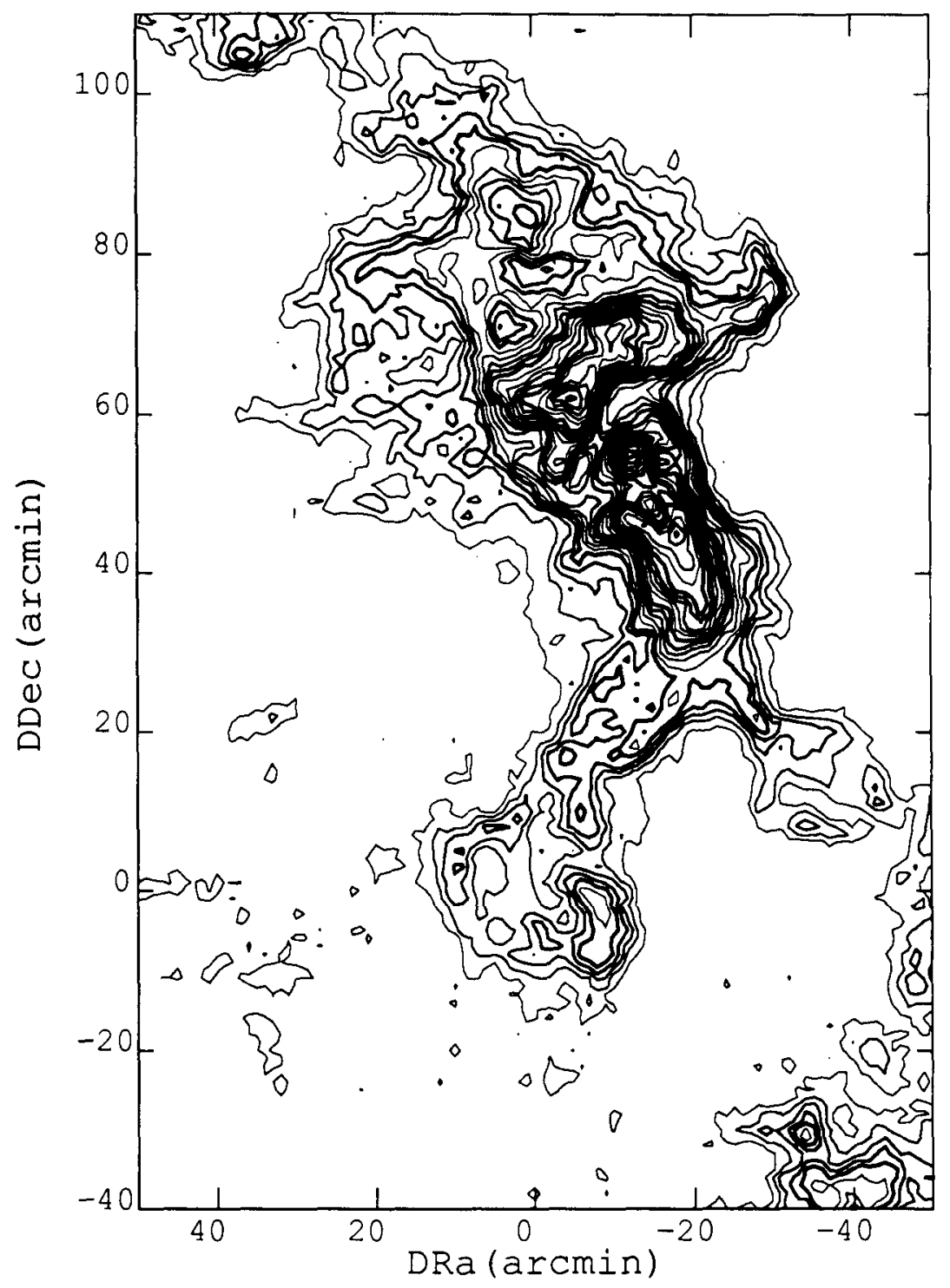

Figure 1: A ${ }^{13} \mathrm{CO}$ contour map showing the emission integrated from $\mathrm{V}_{\mathrm{LSR}}=9 \mathrm{~km} \mathrm{~s}^{-1}$ to $V_{\text {LSR }}=11 \mathrm{~km} \mathrm{~s}^{-1}$. The contour interval is $1 \mathrm{Kkm} \mathrm{s}^{-1}$ and ranges from 1 to $20 \mathrm{Kkm} \mathrm{s}^{-1}$. All co-ordinates are arc-minute offsets from $\alpha=05^{\mathrm{h}} 45^{\mathrm{m}} 0.1^{\mathrm{s}}$ and $\delta=-0^{\circ} 47^{\prime} 00^{\prime \prime}$. Note the bubbles near the bottom of the figure and near the right edge. The reflection nebulae NGC2071 and NGC2068 are located in the main concentration above the center of the figure. 
cloud in the north contains two separate star forming centers; NGC2023 and NGC2024 (the radio source Orion $B$ ) and a separate region of lower mass star formation associated with the reflection nebulae NGC2068 and NGC2071 to the north. In ${ }^{13} \mathrm{CO}$ emission, the cloud is highly fragmented into clumps, filaments, sheets, and partial shell structures, down to a size scale of $0.2 \mathrm{pc}$. Figure 1 shows a map of ${ }^{13} \mathrm{CO}$ emission in the region containing NGC2068 and NGC2071. South of the reflection nebulae, several cavities with bright walls and relatively empty interiors are seen. North of NGC2071, there are a series of arches of molecular gas which may be fragments of shells or filaments. Several streamers of gas extend from the eastem and western ends of the dense ridge of gas containing NGC2071 and trail off in a direction pointing away from the center of the Ori $O B$ association. Such "wind-swept" structure can also be seen in the cometary globules and clouds west of the main Orion molecular clouds.

The Orion A cloud has a systematic velocity gradient along its length. The mean gas velocity varies from $V_{L S R}=4 \mathrm{~km} \mathrm{~s}^{-1}$ in the south to $V_{L S R}=12 \mathrm{~km} \mathrm{~s}^{-1}$ in the north. There is a smaller amplitude chaotic component to the velocity field superimposed on the large scale motion. The velocity gradient may indicate rotation, or can be interpreted as sheer. It is possible that the northern end of the cloud, which lies close to the older $\mathrm{OB}$ subgroups, and contains some of the youngest ones, has been accelerated by interaction with ionizing radiation and the resulting rocket effect. The positive velocity shift in the north requires that the cloud lies mostly on the far side of the association, which is consistent with the visibility of Barnard's Loop in front of the southem portion of Orion A cloud and with the position of the Orion Nebula in front of the molecular cloud.

Many filaments lace the Orion A cloud; some of the largest ones are several degrees long and are aligned with the major axis of the cloud, including the integral-sign shaped high density ridge containing the $\mathrm{OMCl}$ cloud core lying behind the Orion Nebula. Some smaller filaments lie nearly parallel to the cloud minor axis, are less than $100^{\text {" wide but }}$ over $1^{\circ}$ degree long. Like in Orion $B$, close inspection of the images reveal many cavities and partial shells, suggesting local disruption of the cloud by embedded stellar objects. One prominent 20' radius shell and partial cavity surrounds NGC1999 and the HH 1-2 cloud core lying roughly $1^{\circ}$ south of the Orion nebula. Smaller cavities and shells are superimposed on this structure at different velocities.

In addition to the over $10^{5} \mathrm{M}_{0}$ of gas in the Orion A and B clouds, there are many smaller clouds lying to the west of the main cloud concentration at even lower galactic latitudes. Many of these clouds as well as the entire Orion A cloud have cometary shapes whose axes of symmetry point towards the center of the Orion $\mathrm{OB}$ association. The entire northern portion of the Orion A cloud appears compressed relative its southern part. CS observations can be used to infer that the mean gas density in the "integral" shaped filament is much higher than in the southern portion of the cloud. The cloud shapes and velocity gradients suggest that the large scale structure of the cloud has been determined to a large extent by interaction with the $\mathrm{OB}$ association. Ionizing radiation can dissociate, heat, and ionize the cloud forming both high and low density HII regions. The ablated gas may be further heated by the shocks produced by fast stellar winds and supernova explosions and will be eventually incorporated into hot, high pressure, but low density interior medium of the Orion superbubble, driving its expansion. In return, the high pressure of the hot medium, and ablation by ionizing radiation can drive shock waves into the colder molecular cloud, resulting in compression and acceleration. Lower mass regions may be accelerated more, resulting in the wind swept cometary appearance of some of the clouds.

Compression of the northem portion of Orion A and the westem portion of Orion B may have triggered massive star formation occurring at these sites. If so, the shocks may not be always plane- parailel as suggested by Elmegreen and Lada (1977). If a density enhancement retards the shock velocity relative to the surrounding medium, a conical pinch may compress the medium from all sides, producing a filament pointing toward the point of origin of the shock source. This may be the way the compressed filament at the northern end of the Orion A cloud formed. 


\section{EXCITATION OF CO IN THE ORION A MOLECULAR CLOUD}

Alain Castets and Gilles Duvert at the University of Grenoble have mapped many square degrees in the Orion $\mathrm{A}$ molecular cloud in the $\mathrm{J}=2-1$ transitions of ${ }^{12} \mathrm{CO}$ and ${ }^{13} \mathrm{CO}$ using the 2.5-meter POM-2 antenna located on the Plateau de Bure. Combined with the Crawford Hill $\mathrm{J}=1-0$ observations, this data can be used to analyze the excitation conditions of the $\mathrm{CO}$ molecule in an entire molecular cloud. As described in Castets et al. (1989), there are problems with the model frequently used to analyze $\mathrm{CO}$ data. Usually, the ${ }^{12} \mathrm{CO}$ line is assumed to measure the excitation temperature along the line-of-sight, along which it is assumed to be constant. Since the ${ }^{13} \mathrm{CO}$ line is always fainter, it is assumed to be optically thin and used to determine the column density of the gas, under the assumption that the ${ }^{12} \mathrm{CO}$ and ${ }^{13} \mathrm{CO}$ excitation temperatures are the same and uniform along the line-of-sight. Castets et al. (1989) show that this assumption is wrong over much of the Orion A cloud.

The $\mathrm{J}=2-1$ and $\mathrm{J}=1-0{ }^{13} \mathrm{CO}$ lines are observed to have a nearly identical peak intensity and line shape along $90 \%$ of the lines-of-sight through the Orion A cloud. Since the $\mathrm{J}=2-1 \mathrm{CS}$ line can be detected $\mathrm{T}_{\mathrm{A}}>0.1 \mathrm{~K}$ along most directions, the gas density is likely to be sufficient to thermalize ${ }^{13} \mathrm{CO}$. Castets et al. (1989) conclude that the ${ }^{13} \mathrm{CO}$ lines are optically thick and abandon the assumption that the excitation temperatures of ${ }^{13} \mathrm{CO}$ and ${ }^{12} \mathrm{CO}$ are equal. The observed $\mathrm{J}=1-0{ }^{12} \mathrm{CO} /{ }^{13} \mathrm{CO}$ line ratio is explained by requiring the ${ }^{12} \mathrm{CO}$ emitting layer to have a higher excitation temperature than the ${ }^{13} \mathrm{CO}$ layer. This can occur if clouds are mostly heated from the outside. The penetration depth of the ultraviolet radiation responsible for heating cloud surfaces by means of photoelectrons emitted from grains is similar to the depth into the cloud where ${ }^{12} \mathrm{CO}$ saturates $(\tau=1)$ for a typical 1 to $4 \mathrm{~km} \mathrm{~s}^{-1}$ line width and a gas temperature of order 20 to $30 \mathrm{~K}$. The column density lying in front of the $\tau=1$ surface for the $\mathrm{J}$ $=1-0$ transition of $\mathrm{CO}$, (the penetration depth) is given by

$$
\mathrm{N}_{\tau=l}\left(\mathrm{H}_{2}\right)=\frac{2.4 \times 10^{14} \mathrm{~T}_{\mathrm{rot}} \Delta v\left(\mathrm{~km} \mathrm{~s}^{-1}\right)}{\mathrm{X}_{\mathrm{CO}}\left[1-\exp \left(-\mathrm{hv} / \mathrm{kT}_{\mathrm{ex}}\right)\right]}
$$

Where $X_{C O}$ is the relative abundance of the CO species relative to hydrogen, $T_{\text {rot }}$ is the excitation temperature, and $\Delta v$ is the line width. For $\Delta v=2 \mathrm{~km} \mathrm{~s}^{-1}, T=20 \mathrm{~K}$, and $\mathrm{X}_{\mathrm{CO}}=5 \times 10^{-5}$ (appropriate for ${ }^{12} \mathrm{CO}$ ), this gives $\mathrm{N}_{\tau=1}\left(\mathrm{H}_{2}\right) \approx 4 \times 10^{20} \mathrm{~cm}^{-2}$. For $\mathrm{T}=10 \mathrm{~K}$ and $X_{\mathrm{CO}}=1 \times 10^{-6}$ (for ${ }^{13} \mathrm{CO}$ ) with the same line-width, $\mathrm{N}_{\tau=1}\left(\mathrm{H}_{2}\right)=9 \times 10^{21} \mathrm{~cm}^{-2}$ Thus the ${ }^{12} \mathrm{CO}$ lines probe the externally heated cloud surface while the ${ }^{13} \mathrm{CO}$ lines probe the shielded cloud interior.

The ${ }^{13} \mathrm{CO}$ lines appear to be optically thin the immediate vicinity of luminous embedded sources, such as irc2 in the Orion A cloud core. Here, the gas is hot and the CO population is spread over many levels in the rotation ladder. The radius of influence of an embedded heating source of luminosity $\mathrm{L}_{100}$ (measured in units of $100 \mathrm{~L}_{\mathrm{O}}$ ) is given by $R(T<10 \mathrm{~K})=0.4 \mathrm{~T}_{10}^{-5 / 2} \mathrm{~L}_{100}^{1 / 2}$ (parsecs) where $\mathrm{T}$ is in units of $10 \mathrm{~K}$. In a typical GMC, there are only several dozen sources with luminosities above $100 \mathrm{~L}$, so only a small fraction of the mass and volume of a GMC can be intemally heated. The rest of the interior of molecular clouds are probably heated by cosmic rays, dissipation of turbulence or shock waves, or possibly by ambipolar diffusion and remain cold.

\section{CAN NON-IONIZING UV RADIATION PRODUCE BUBBLES AND TURBULENCE?}

The superthermal line widths of spectral lines in molecular clouds has been a long standing mystery. In order to maintain the supersonic turbulence for more than a dissipation time scale $\tau_{\text {dis }}$, which is the order of 10 free fall-times $\left(10^{6}\right.$ to $10^{7}$ years), some mechanism must supply kinetic energy at a rate $M_{\text {cloud }} \Delta V_{\text {turb }}^{2} / \tau_{\text {dis }}$ where $\Delta V_{\text {turb }}$ is the line width and $\tau_{\text {dis }}$ is the dissipation time scale. External agents are inefficient as a source of energy input since the large density and temperature difference between the molecular cloud and the surrounding 
medium provides an impedance mismatch. Most of the energy of supernova explosions, stellar winds from main sequence stars, or radiation is thought to couple into the intercloud medium or to accelerate the clouds as a whole. A source of energy which resides inside the cloud is more efficient.

With the discovery of bipolar outflows and the recognition that virtually all young stellar objects undergo a period of energetic mass loss, it has become evident that young stars are a source of the energy to support the turbulence (Bally, 1982). If clouds had smooth internal structure, flows would be capable of efficiently injecting kinetic energy into a cloud. However, the observed fragmented structure implies that the energy may be more efficiently coupled to the intercloud medium than to the dense $\mathrm{CO}$ emitting gas. The early calculations on support of clouds by outflows neglected the inefficiency of the coupling of the flow energy to a cloud which is highly fragmented on a scale smaller than the characteristic size of the flow. In this case, the flow is expected to blow out of the dense cloud, and its energy will not couple directly into the dense gas.

Although it is possible that some bubbles and partial shells are fossil outflows, their large size and lack of bipolarity suggest that some other mechanism is at work. Main sequence stars can be found near the centers of some bubbles and partial shells. The partial rings surrounding NGC1999 are nearly concentric with the bright reflection nebulosity, suggesting that the stars producing the scattered light may also be responsible for the large scale structure in the gas. Although winds produced during both the pre-main- sequence and main-sequence life of a star may help inflate a bubble, it is intriguing to to investigate the possible role of non-ionizing radiation.

Soft UV radiation is responsible for heating the surface layers of clouds. In regions near B or A type stars, the radiation field can be orders of magnitude stronger than in average locations in interstellar space. Tielens and Hollenbach (1985) and Hollenbach (1988) have shown that the temperature of the boundary layer of a cloud can be raised to hundreds of degrees by a combination of photoelectric and other heating processes. As discussed above, there is evidence for an increase in the average temperature of the $\mathrm{CO}$ emitting layer relative to the shielded cloud interior. Heated gas at the cloud surface can stream away from the cloud at a velocity of a few $\mathrm{km} / \mathrm{sec}$, resulting in a reaction force on the cloud, analogous to the rocket effect produced by ionizing radiation. The effect of the non-ionizing component is expected to be similar in nature but milder in amplitude. A column density $\mathbf{N}$ of gas can be accelerated to a velocity $\mathrm{V}$ by a source of soft $\mathrm{UV}$ of luminosity $\mathrm{L}_{*}$ in a time $\tau$ given by $\tau=2 \pi \mathrm{r}^{2} \mu \mathrm{m}_{\mathrm{H}} \mathrm{Nv}^{2} / \mathrm{X}_{\mathrm{UV}} \varepsilon \mathrm{L}_{*}$ which for $\mathrm{N}=10^{21} \mathrm{~cm}^{-2}, \mathrm{r}=10 \mathrm{pc}, \mathrm{L}_{*}=100 \mathrm{~L}_{\mathrm{O}}$, and a UV fraction of $10 \%$, and an overall UV heating efficiency of $0.1 \%$ implies that a velocity of 1 $\mathrm{km} \mathrm{s}^{-1}$ can be reached in $10^{5}$ years. Soft UV might produce some shells and bubbles, and together with outflows, and externally generated shocks, may contribute kinetic energy required to support the supersonic cloud line widths.

\section{REFERENCES}

Bally, J 1982 Ann.N.Y.Acad.Sci.,395,191.

Bally, J., Langer, W.D, Wilson, R.W. and Stark, A.A. 1987 Ap.J.(Letters), 312,L45.

Bregman, J. 1980 Ap.J.,236,577.

Castets, A., Duvert, G., Bally, J., Wilson, R.W., Langer, W.D., and Dutrey, A. Astron.Astrophys.,(in preparation). 
Cowie, L.L., Songaila, A., and York,D.G. 1979 Ap.J.,230,469.

Elmegreen, B.G. and Lada, C.J. 1977 Ap.J.,214,725.

Goudis, C. 1982 The Orion Complex: A Case Study of Interstellar Matter.

(Dordrecht: D. Reidel).

Sivan, J.P. 1974 Astron.Astophys.(Suppl.),16,163.

Tielens, A.G.G.M. and Hollenbach, D.J. 1985 Ap.J.,291,722.

Hollenbach, D.J. 1988 I.A.U. Symposium \#135, Interstellar Dust, ed. A.G.G.M. Tielens and L. Allamandola.

\section{Discussion:}

FRANCO: Photodissociating radiation (and perhaps even radiation pressure on dust grains) may add some energy to the case of the Pleiades.

BALLY: Yes I agree. The main point I want to make is that the TOTAL heating rate (sum of photodissociation, photoelectric, etc.) may have important dynamical consequences for the structure and kinematics of clouds. Hot gas in the boundary layer will expand away form the cloud surface, and the resulting "rocket" effect can accelerate the cloud. This may produce bubbles such as seen in Orion, surrounding the Pleiades. In enviroments such as Orion, where there are many later than B3 stars, this mechanism may dominate input from outflows in providing cloud support.

MÜNCH: The photodissociation of $\mathrm{H}_{2}$ should occur in a narrow range fairly near the "surface" of a cloud $\left(\tau_{\text {opt }} \sim 1\right)$. In order to maintain the cloud heated we badly need cosmic rays.

BALLY: The UV penetration depth is roughly $\tau_{U V} \sim 1 / 3$ to $1 / 5 \tau_{\text {opt }}$. The dissociation of $\mathrm{CO}$ occurs within this region. Since $\tau_{\text {opt }}$ corresponds to a hydrogen column density of $10^{21} \mathrm{~cm}^{-2}$, the ${ }^{12} \mathrm{CO}$ line saturates well within the region where UV heating plays a dominant role (via photoelectric heating and $\mathrm{H}_{2}$ dissociation heating). Visual and near IR photons, however, can still penetrate to $\tau_{\text {opt }} \sim$ few with sufficient efficiency to dominate cosmic ray heating, and this "long wavelength" heating will be substantial in environments where the ambient radiation field is stronger than the average value. The interior of the cloud can be heated to 5 to $10 \mathrm{~K}$ required to explain the observed intensity of ${ }^{13} \mathrm{CO}$ by either cosmic-rays or the dissipation of supersonic turbulence which dominates the millimeter wavelenght line-widths. The proposed rocket effect produced by the ablation of the hot surface layers of the cloud may sustain this turbulence. 